\title{
Efficacy of lactobacilli to normalize production of corticosterone induced by unpleasant handling of broilers
}

\author{
A. Meimandipour ${ }^{1}$, A. Soleimanifarjam ${ }^{2}$, M. Hair-Bejo ${ }^{3}$, M. Shuhaimi ${ }^{4}$, K. Azhar $^{2}$, \\ L. Nateghi ${ }^{5}$, B. Rasti ${ }^{1}$ and A.M. Yazid ${ }^{1 \#}$ \\ ${ }^{1}$ Department of Food Technology, Faculty of Food Science and Technology \\ ${ }^{2}$ Department of Animal Science, Faculty of Agriculture \\ ${ }^{3}$ Department of Veterinary Pathology and Microbiology, Faculty of Veterinary \\ ${ }^{4}$ Halal Products Research Institute, Universiti Putra Malaysia, 43400 Selangor, Malaysia \\ ${ }^{5}$ Faculty of Food Science and Technology, Islamic Azad University, Varamin-Pishva Branch, Varamin, Iran
}

\begin{abstract}
A study was conducted to investigate the effect of two Lactobacillus strains on hypothalamus pituitary adrenal (HPA) axis activity induced by supposedly unpleasant handling (UH) of broiler chickens. The three treatments were: (1) non-handled chicks fed basal diet (control); (2) unpleasantly handled-chicks fed basal diet (UH-BD) and (3) unpleasantly handled-chicks fed basal diet supplemented with the probiotic, lactobacilli (UH-BDL). Chicks were exposed to UH from days 1 to 21. Treatment UH-BDL received probiotics in their diet for the whole experimental period. Blood corticosterone (CS) concentrations were monitored at 14, 28, 35 and 42 days of age and selected caecal bacterial groups were enumerated in 14-day old birds. Unpleasant handling significantly increased blood CS concentrations at 14 and 28 days of age. Blood CS concentration decreased with age over the course of the experimental period. Lactobacillus supplementation did not reduce blood CS concentration in broilers in the UH-BDL treatment. Moreover, the UH treatment did not alter caecal bacterial numbers in the 14-day old broilers while the probiotic numerically increased total anaerobes and lactobacilli. Overall, it seems as if the efficacy of lactobacilli to modulate stress-related high HPA-axis activity depends on gastrointestinal tract microbial alteration. However, Lactobacillus salivarius subsp. salicinius JCM 1230 and L. agilis JCM 1048 were able to reestablish a proper microbial balance in the caecum of the chickens.
\end{abstract}

Keywords: Caecal microflora, chickens, hypothalamus pituitary adrenal axis, physical contact, probiotic

\#Corresponding author. E-mail: myazid@food.upm.edu.my

\section{Introduction}

Gastrointestinal tract (GIT) microbiota is crucial to the well-being of animals. There are 10 times more microbiota than enterocytes cells, some of which have important health functions. In chickens, intestinal bacterial colonization begins soon after hatch, and continues to be established by week 2 post-hatch (AmitRomach et al., 2004). It has been reported that the GIT microflora cooperate in the development of the hypothalamus-pituitary-adrenal (HPA) axis (Sudo et al., 2004), which is necessary for the normal stress response. On the other hand, stressful experience can disturb the balance of the intestinal microbiota and lead to the excessive growth of pathogens while decreasing the proportion of beneficial bacteria such as lactobacilli and bifidobacteria (Fuller, 1999; Lan et al., 2004; Selig \& Patterson, 2004; Lutgendorff et al., 2008). Thus, any changes in the GI tract microbiota due to stress may negatively influence HPA axis activities, for example, Gareau et al. (2007) showed that maternal separation in mice increased blood corticosterone level as compared to the control. However, the addition of probiotics to the diet of stressed mice decreased the elevated level of blood corticosterone to that in unstressed mice, suggesting normalized activity of the HPA axis. Probiotics stimulate different cells in the immune system to produce cytokines, and also regulate HPA-axis activity via the enteric nerves.

Commercial broilers reared under intensive and artificial conditions experience fear over the course of their life. Farm animals that were handled adversely became highly fearful of human beings and had elevated basal concentrations of corticosteroids (Hemsworth \& Gonyou, 2000). To the best of our knowledge the influence of probiotics on HPA axis activity and CS secretion induced by unpleasant handling (UH) in broiler chickens during the first three weeks of life when the GIT bacterial community and nervous system 
are under development has not been investigated. Therefore, it can be hypothesized that two strains of Lactobacillus salivarius subsp. salicinius JCM 1230 and Lactobacillus agilis JCM 1048 can reduce the enhanced level of CS due to UH, at this period of time.

\section{Materials and Methods}

Lactobacillus salivarius subsp. salicinius JCM 1230 and L. agilis JCM 1048 obtained from JCM (Japan collection of micro-organisms) were routinely grown in MRS broth (Oxoid, Basingstoke, UK) at $37^{\circ} \mathrm{C}$. Overnight MRS cultures were harvested by centrifugation $\left(3640 \times \mathrm{g}\right.$ for $15 \mathrm{~min}$ at $\left.4{ }^{\circ} \mathrm{C}\right)$ and washed twice with a sterile phosphate buffer saline solution, $\mathrm{pH}$ 7.2. The cells were then resuspended in a $10 \%$ skimmed milk solution (wt/vol) and freeze-dried for three days. To ensure the maximum viability of the preparation, the freeze-dried samples were stored at $4{ }^{\circ} \mathrm{C}$ and added daily to the feed $\left(10^{7} \mathrm{CFU} / \mathrm{strain} / \mathrm{g}\right)$. At two weeks intervals the freeze dried culture was counted to ensure the maintenance of the concentration of viable lactobacilli in the feed.

All the procedures and in particular the unpleasant handling were approved and monitored by the Animal Care and Use Committee of the University, Putra Malaysia to ensure that the experiment would not conflict with animal welfare and with the safety of rearing the experimental animals. A total of 105 day-old male Cobb broilers were obtained from a commercial hatchery. On arrival, the chicks were wing-banded, individually weighed and assigned to 15 experimental units of five replicates with the following treatments: (1) Five experimental units (35 chicks) were fed a basal diet and received normal routine husbandry (control); (2) Five experimental units (35 chicks) were fed the basal diet and received UH (UH-BD); (3) Five experimental units (35 chicks) were fed the basal diet supplemented with $10^{7}$ CFU of each Lactobacillus strain per gram of feed and received UH (UH-BDL). The chicks were placed in battery cages inside an

Table 1 Composition of the experimental diets $(\mathrm{g} / \mathrm{kg})$

\begin{tabular}{lcc}
\hline & $\begin{array}{c}\text { Starter diet } \\
(0-21 \text { Days })\end{array}$ & $\begin{array}{c}\text { Grower diet } \\
(22-42 \text { Days })\end{array}$ \\
\hline Ingredients & & \\
Maize & 612 & 645 \\
Soya bean meal (48) & 283 & 245 \\
Palm oil & 20 & 35 \\
Fish meal & 50 & 40 \\
Calcium carbonate & 12 & 12 \\
Dicalcium-phosphate & 13 & 13 \\
Vitamin premix & 2.5 & 2.5 \\
Mineral premix & 2.5 & 25 \\
Methionine & 2 & 2 \\
Calculated composition & & \\
Crude protein & 215 & 193 \\
ME (MJ/kg) & 12.5 & 12.9 \\
Lysine & 11.6 & 5.4 \\
Methionine & 5.7 & 9.6 \\
Calcium & 10 & 4.4 \\
Nonphytate phosphorus & 4.6 & 1.3 \\
Sodium & 1.3 &
\end{tabular}

\footnotetext{
${ }^{1}$ Provided per kg of diet: $4500 \mathrm{IU}$ vitamin $\mathrm{A} ; 1000 \mathrm{IU}$ vitamin $\mathrm{D}_{3} ; 50 \mathrm{mg}$ vitamin $\mathrm{E} ; 1.5 \mathrm{mg}$ vitamin $\mathrm{K}$; $0.02 \mathrm{mg}$ vitamin $\mathrm{B}_{12} ; 3 \mathrm{mg}$ vitamin $\mathrm{B}_{2} ; 5 \mathrm{mg}$ pantothenic acid; $20 \mathrm{mg}$ niacin; $150 \mathrm{mg}$ choline chloride; $0.5 \mathrm{mg}$ folic acid.

${ }^{2}$ Provided per kg of diet: $40 \mathrm{mg}$ zinc; $80 \mathrm{mg}$ iron; $0.8 \mathrm{mg}$ iodine; $60 \mathrm{mg}$ manganese; $8 \mathrm{mg}$ copper; $0.2 \mathrm{mg}$ selenium; 0.4 mg cobalt.
} 
environmentally controlled chamber and the diets (antibiotic-free) were formulated, based on maize and soyabean meal to meet NRC requirements (1994), in a 2-phase feeding programme up to 42 days of age. The composition of the basal diets is shown in Table 1.

The unpleasant handling procedure applied in this study was a modification of that described by Zulkifli \& Siti Nor Azah (2004). Briefly: Once daily from day 1 to day 21 the chickens in the UH treatment were transferred from their crates to a separate room. They were suspended by their legs in a group while being swung gently for a period of $30 \mathrm{~s}$. Following the UH treatment, the birds were returned to their crates inside the chamber.

At day 14, five birds from different experimental units in each treatment were randomly sacrificed by cervical dislocation. Their caeca were aseptically collected, tied on the open sides, placed in a sterile plastic bag on ice and immediately transferred to the laboratory. Caecal digesta were squeezed into an empty preweight sterile bottle, homogenized in a phosphate buffer saline $(10 \% \mathrm{wt} / \mathrm{vol})$ and serially diluted to $10^{-10}$. Duplicate plates for each dilution and selected bacterial group were then inoculated and incubated at $37^{\circ} \mathrm{C}$, aerobically or in anaerobic conditions (anaerobic jar with Anaerocult; Merck). Total aerobes, total anaerobes and lactobacilli were enumerated using nutrient agar, Wilkins-Chalgren agar and MRS, respectively. After a $48 \mathrm{~h}$ incubation, colonies were counted and characterized based on their appearance, Gram reaction, microscopic characteristics and limited biochemical profiles (Wang \& Gibson 1993).

At 14, 28, 35 and 42 days of age, five chicks from different experimental units in each treatment were randomly selected and blood samples were collected from the wing vein for determining plasma concentrations of CS (EDTA, anticoagulant). To minimize the influence of the sampling procedure on the circulating levels of corticosterone, the birds were taken from the cages one by one and a blood sample was collected from each bird within $40 \mathrm{~s}$ (Lagadic et al., 1990). Blood samples for the hormone assay were centrifuged and the serum separated and stored at $-20^{\circ} \mathrm{C}$ until assayed. The plasma concentration of CS was measured using a commercial RIA kit (MP Biomedicals, Irvine, CA; Severson et al., 1978). The concentration of CS in serum samples was calculated from a standard curve and expressed in nanograms per millilitre (ng/mL).

The main objective of the present study was to investigate the effects of two independent variables, i.e. $\mathrm{UH}$ and lactobacilli supplementation, on the CS concentration in blood plasma and bacterial log counts as response variables. In this study a Completely Randomized Block Design (CRBD) was applied to run the treatment based on the independent variable assigned in this study. The One sample t-test was used to determine significant differences within a sample or group. A univariate one-way analysis of variance (ANOVA) of SAS (1994) was applied to evaluate significant differences between the treatments. Significant differences between the means of the treatments were separated using the Duncan's Multiple Range test at $\mathrm{P}<0.05$.

\section{Results}

Table 2 Mean blood corticosterone concentration $(\mathrm{ng} / \mathrm{mL})$ of broiler chickens exposed to unpleasant handling (UH) and fed a diet supplemented with Lactobacillus over the course of the experiment

\begin{tabular}{|c|c|c|c|c|c|}
\hline \multirow{2}{*}{ Items } & \multicolumn{3}{|c|}{ Treatments } & \multirow[b]{2}{*}{ Pooled SD } & \multirow[b]{2}{*}{ P-value } \\
\hline & Control & UH-BD & UH-BDL & & \\
\hline \multicolumn{6}{|c|}{ Corticosterone concentration (ng/mL) } \\
\hline Day 14 & $5.90^{\mathrm{b}}$ & $8.08^{a}$ & $7.52^{\mathrm{a}}$ & 0.67 & 0.003 \\
\hline Day 28 & $1.97^{\mathrm{b}}$ & $5.90^{\mathrm{a}}$ & $5.67^{\mathrm{a}}$ & 0.59 & 0.001 \\
\hline Day 35 & 1.23 & 1.40 & 1.49 & 0.19 & 0.219 \\
\hline Day 42 & 0.21 & 0.20 & 0.22 & 0.03 & 0.574 \\
\hline
\end{tabular}

\footnotetext{
Means within rows with different superscripts are significantly different $(\mathrm{P}<0.05)$.

Control - Birds subjected to the normal human contact and fed the basal diet;

UH-BD - Birds subjected to unpleasant handling from day 1 to day 21 and fed the basal diet;

UH- BDL - Birds subjected to unpleasant handling from day 1 to day 21 and fed the basal diet containing lactobacilli.
} 
The influence of dietary Lactobacillus supplementation and UH on the concentration of blood plasma CS over the course of the experiment is shown in Table 2. At days 14 and 28 both UH-BD and UH-BDL treated broilers showed higher $(\mathrm{P}<0.01)$ levels of corticosterone than the broilers in the control. The concentration of blood plasma CS decreased ( $\mathrm{P}<0.01$ ) with age from 14 to 42 days of age (Table 3). The results of the UH treatment and lactobacilli supplementation on the selected caecal microbial groups are presented in Figure 1. There were no differences in the number of total aerobes, total anaerobes and lactobacilli between treatment groups at 14 days of age. However, broilers in the UH-BDL treatment showed a numerically higher $(\mathrm{P}<0.05)$ number of total anaerobes and lactobacilli compared to the other groups.

Table 3 Mean blood corticosterone concentration (ng/mL) over the course of the experiment

\begin{tabular}{lcccccc}
\hline \multicolumn{7}{c}{ Age } \\
\hline Items & Day 14 & Day 28 & Day 35 & Day 42 & Pooled SD & P-value \\
\hline Control & $5.90^{\mathrm{a}}$ & $1.97^{\mathrm{b}}$ & $1.23^{\mathrm{b}}$ & $0.21^{\mathrm{c}}$ & 0.44 & 0.001 \\
UH-BD & $8.08^{\mathrm{a}}$ & $5.90^{\mathrm{b}}$ & $1.40^{\mathrm{c}}$ & $0.20^{\mathrm{d}}$ & 0.45 & 0.001 \\
UH-BDL & $7.52^{\mathrm{a}}$ & $5.67^{\mathrm{b}}$ & $1.49^{\mathrm{c}}$ & $0.22^{\mathrm{d}}$ & 0.48 & 0.001 \\
\hline
\end{tabular}

Means within rows with different superscripts are significantly different $(\mathrm{P}<0.05)$.

Control - Birds subjected to the normal human contact and fed the basal diet;

UH-BD - Birds subjected to unpleasant handling from day 1 to day 21 and fed the basal diet;

UH- BDL - Birds subjected to unpleasant handling from day 1 to day 21 and fed the basal diet containing lactobacilli.

\section{Discussion}

The results from the current experiment indicated clearly that subjecting broilers to unpleasant handling for 21 consecutive days profoundly increased their plasma CS concentration. Stressed chicks normally exhibit elevated plasma levels of CS, whether treated with adrenocorticotropin (ACTH) (Minozzi et al., 2008), CS (Post et al., 2003) or exposed to different stressors including tonic immobility (Jones et al., 2005), high temperature (Lin et al., 2006) or feed restriction (Sandilands et al., 2006). Blood CS concentration is an indicator of HPA-axis activity. Stress induces the release of corticotropin-releasing hormone (CRH) in the hypothalamus which subsequently leads to the secretion of ACTH from the pituitary gland. Adrenocorticotropin causes the secretion of glucocorticoids from the adrenal glands. The results of the current study also demonstrated that plasma CS concentration declined with age from 14 to 42 days of age. It has been shown that prolonged adrenal stimulation leads to its exhaustion and reduced circulating levels of CS (Puvadolpirod \& Thaxton, 2000). However, Freeman et al. (1979) reported that this decline was due to habituation in response to repeated stimulus.

The addition of lactobacilli to the diet of unpleasantly handled birds did not reduce blood CS concentrations over the course of the experiment. This result was in contrast with those of a previous study (Gareau et al., 2007) which showed that the addition of probiotics to the diet decreased elevated blood CS concentrations of stressed neonatal rats and that this coincided with a five fold decrease in Lactobacillus species in the colon of stressed rats compared to their unstressed counterparts. Furthermore, restraint stress in germ-free mice increased the HPA-axis activity compared to conventional mice, which was reversed by the administration of probiotic bacteria to the germ free group (Sudo et al., 2004). The findings from these studies clearly demonstrated a relationship between the GIT microbial community, particularly the beneficial bacteria and HPA-axis development which might be disrupted by the detrimental effects of stress. The authors reported that some components of the bacterial cell wall may induce immune cells to secrete cytokines which consequently affect HPA-axis activity. The second proposed mechanism was probably mediated by the enteric nerves. In the present study, although duodenal, ileal and caecal lactate concentrations together with caecal butyrate level were significantly $(\mathrm{P}<0.05)$ decreased in broilers on the UH-BD diet compared to the control and UH-BDL groups at 14 and 21 days of age (unpublished data), it did not have any influence on lactobacilli and other selected caecal microbial populations at 14 days of age. 


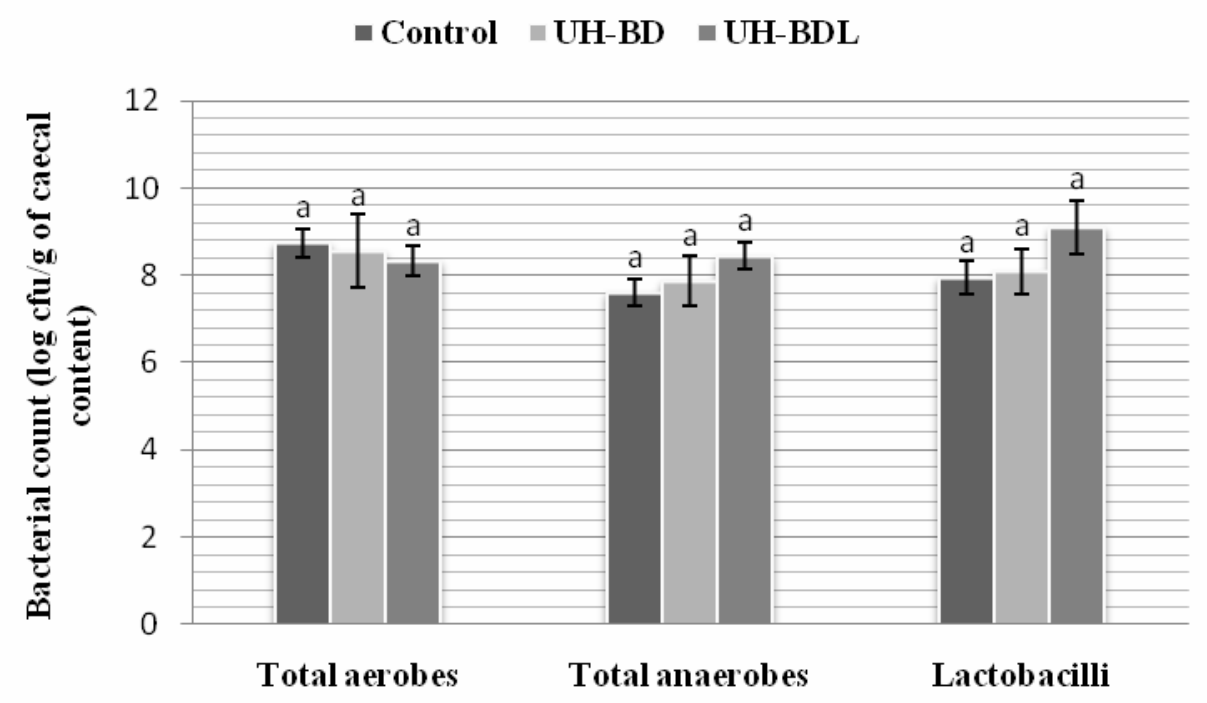

Figure 1 Caecal bacterial population means of broiler chicken exposed to unpleasant Handling and fed a diet supplemented with Lactobacillus at 14 days of age.

Bars are expressed as mean log10 bacterial colony counts (per gram wet caecal digesta) of duplicate determination. Error bars indicate standard deviation of the means. Different letters indicate statistically significant differences $(\mathrm{P}<0.05)$.

Control - Birds subjected to the normal human contact and fed basal diet;

UH-BD - Birds subjected to unpleasant handling from day 1 to day 21 and fed basal diet;

UH- BDL - Birds subjected to unpleasant handling from day 1 to day 21 and fed basal diet containing lactobacilli.

It should be emphasized that caecal bacterial metabolic end-products such as SCFAs may not necessarily reflect the population level of bacteria in the caecum (Ichikawa et al., 1999). In fact, carbohydrate degradation to produce SCFAs are interactive processes among various bacteria (Macfarlane \& Gibson, 1995), in which only a minor change in the GIT microbiota could profoundly alter its overall metabolism. Therefore, it can be concluded that the unpleasant handling used in the current study was not too adverse to negatively affect the population levels of bacteria. This might be the reason why the addition of lactobacilli did not decline HPA-axis activity and subsequently blood CS concentration. Furthermore, host intrinsic differences in response to stress and type of applied stress should not be overlooked. Dhabhar et al. (1997) demonstrated that by giving both a single prolonged $(4 \mathrm{~h})$ stress and stress sessions repeated over a period of 10 days to three different strains of rats, strain FISCHER 344 showed a greater $(\mathrm{P}<0.05)$ activation of the HPA-axis and higher blood CS concentrations compared with the other strains (Sprague-Dawley and Lewis). The authors reported that the FISCHER 344 strain showed no habituation or adaptation to CS stress response during both single and repeated stress whereas the other strains did. In the present study the birds were exposed to prolonged UH from one to 21 days of age. However, broilers in both the UH and the control groups showed habituation to CS handling response over the course of the experiments which might explain the lack of any changes in HPA-axis activity due to lactobacilli supplementation.

The selected caecal microbial populations were not different $(\mathrm{P}<0.05)$ between the control and the UH treatment groups. Previous study by Putsakum et al. (2007) demonstrated no difference in the intestinal microbial populations between ACTH-treated and control birds, whereas in other studies environmental factors, such as stressful conditions influenced bacterial colonization of the gastrointestinal tract (Lan et al., 2004; Selig \& Patterson, 2004; Gareau et al., 2007). As mentioned earlier, type and severity of stress and host intrinsic factors might explain the controversy among these reports. In agreement with our previous in vitro study (Meimandipour et al., 2009) Lactobacillus supplementation numerically increased total anaerobes and lactobacilli colonies in broilers in the UH-BDL treatment as compared with their UH-BD and control counterparts. Probiotic bacteria via competition for nutrient and receptor site, production of SCFAs and other metabolic end products are capable of re-establishing the proper intestinal microflora and beneficially control the GIT ecosystem. 


\section{Conclusions}

The combined findings of the present study suggested that the beneficial effects of probiotics on normalizing HPA-axis activities seem to be dependent on the alteration level of bacterial population due to stress or the lack of bacteria in germ free animals. Furthermore, some factors such as host intrinsic differences, type and intensity of stress, animal adaptation to stress and probiotic strains would probably influence the efficacy of probiotics to normalize enhanced HPA-axis activity due to stressful conditions. In the current study, a UH regime of $30 \mathrm{~s}$ daily in the first three weeks of life was ineffective in altering the chicken GIT microbial population, whereas lactobacilli beneficially altered the GIT bacterial community.

\section{References}

Amit-Romach, E., Sklan, D. \& Uni, Z., 2004. Microbiota ecology of the chicken intestine using 16S ribosomal DNA primers. Poult. Sci. 83, 1093-1098.

Dhabhar, F.S., McEwen, B.S. \& Spencer, R.L., 1997. Adaptation to prolonged or repeated stress comparison between rat strains showing intrinsic differences in reactivity to acute stress. Neuroendocrinology 65, 360-368.

Freeman, B.M., Manning, A.C. \& Flack, I.H., 1979. Habituation by the immature fowl in response to repeated injections of corticotrophin. Br. Poult. Sci. 20, 391-399.

Fuller, R., 1999. Probiotics for farm animals. In: Probiotics: A Critical Review. Ed. Tannock, G.W., Horizon Scientific Press, Wymondham, England. pp. 15-22.

Gareau, M.G., Jury, J., MacQueen, G., Sherman, P.M. \& Perdue, M.H., 2007. Probiotic treatment of rat pups normalises corticosterone release and ameliorates colonic dysfunction induced by maternal separation. Gut 56, 1522-1528.

Hemsworth, P.H. \& Gonyou, H.W., 2000. Human contact. In: Animal Welfare. Eds Appleby, M.C. \& Hughes, B.O., CAB International, Wallingford, UK. pp. 205-217.

Lagadic, H., Faure, J.M., Mills, A.D. \& Williams, J.B., 1990. Effects of blood sampling on plasma concentration and glucose in laying hens caged in groups. Br. Poult. Sci. 31, 823-829.

Ichikawa, H., Kuroiwa, T., Inagaki, A., Shineha, R., Nishihira, T., Satomi, S. \& Sakata, T., 1999. Probiotic bacteria stimulate gut epithelial cell proliferation in rat. Dig. Dis. Sci. 44, 2119-2123.

Jones, R.B., Marin, R.H. \& Satterlee, D.G., 2005. Adrenocortical responses of Japanese quail to a routine weighing procedure and to tonic immobility induction. Poult. Sci. 84, 1675-1677.

Lan, P.T.N., Sakamoto, M. \& Benno, Y., 2004. Effects of two probiotic lactobacillus strains on jejunal and cecal microbiota of broiler chicken under acute heat stress condition as revealed by molecular analysis of 16S rRNA genes. Microbiol. Immunol. 48, 917-929.

Lin, H., Decuypere, E. \& Buyse, J., 2006. Acute heat stress induces oxidative stress in broiler chickens. Comp. Biochem. Physiol. Mol. Integr. Physiol. 144, 11-17.

Lutgendorff, F., Akkermans, L.M. \& Soderholm, J.D., 2008. The role of microbiota and probiotics in stressinduced gastro-intestinal damage. Cur. Mol. Med. 8, 282-298.

Macfarlane, G.T. \& Gibson, G.R., 1995. Microbiological aspects of short chain fatty acid production in the large bowel. In: Physiological and Clinical Aspects of Short Chain Fatty Acid Metabolism, Eds Cummings, J.H., Rombeau, J.L. \& Sakata, T., Cambridge University Press, Cambridge, UK. pp. 87-105.

Meimandipour, A., Shuhaimi, M., Hair-Bejo, M., Azhar, K., Kabeir, B.M., Rasti, B. \& Yazid, A.M., 2009. In vitro fermentation of broiler cecal content: The role of lactobacilli and $\mathrm{pH}$ value on the composition of microbiota and end products fermentation. Lett. Appl. Microbiol. 49, 415-420.

Minozzi, G., Guemene, D., Couty, M., Gourichon, D., Minvielle, F. \& Pinard-van der Laan, M.H., 2008. circulating corticosterone reaction to restraint and adrenocorticotropin hormone administration in white leghorns selected for immune response traits. Poult. Sci. 87, 2225-2230.

NRC, 1994. Nutrient Requirements of Poultry. National Academy Press, Washington, D.C., USA.

Post, J., Rebel, J.M.J. \& ter Huurne, A.A.H.M., 2003. Physiological effects of elevated plasma corticosterone concentrations in broiler chickens. An alternative means by which to assess the physiological effects of stress. Poult. Sci. 82, 1313-1318.

Putsakum, M., 2007. The relationship of diet, stress, intestinal nitric oxide production and intestinal microflora in chickens. PhD thesis. Mississippi State University, USA. 
Puvadolpirod, S. \& Thaxton, J.P., 2000. Model of physiological stress in chickens 1. Response parameters. Poult. Sci. 79, 363-369.

Sandilands, V., Tolkamp, B.J., Savory, C.J. \& Kyriazakis, I., 2006. Behaviour and welfare of broiler breeders fed qualitatively restricted diets during rearing: Are there viable alternatives to quantitative restriction? Appl. Anim. Behav. Sci. 96, 53-67.

SAS, 1994. SAS/STAT User's Guide. Release 6.08 ed. SAS Institute Inc., Cary, N.C., USA.

Selig, K.B. \& Patterson, J.A., 2004. Changes in intestinal microbiota and ileal susceptibility to pathogen attachment in broilers subjected to $24 \mathrm{hr}$ heat stress. American Society of Animal Science, American Society of Dairy Science and Poultry Science Annual meeting. St. Louis, MO. July 26-29.

Severson, J.A., Fell, R.D. \& Griffith, D.R., 1978. Adrenocortical function in response to myocardial necrosis in exercise-trained rates. J. Appl. Physiol. 44, 104-108.

Sudo, N., Chida, Y., Aiba, Y., Sonoda, J., Oyama, N., Yu, X.N., Kubo, C. \& Koga, Y., 2004. Postnatal microbial colonization programs the hypothalamic-pituitary-adrenal system for stress response in mice. J. Physiol. 558, 263-275.

Wang, X. \& Gibson, G.R., 1993. Effects of the in vitro fermentation of oligofructose and inulin by bacteria growing in the human large intestine. J. Appl. Bacteriol. 75, 373-380.

Zulkifli, I. \& Siti Nor Azah, A., 2004. Fear and stress reactions and the performance of commercial broiler chickens subjected to regular pleasant and unpleasant contacts with human being. Appl. Anim. Behav. Sci. 88, 77-87. 